\title{
Efeitos das Técnicas Respiratórias Não Invasivas na Melhora da Função Pulmonar de Cardiopatas: Uma Revisão Integrativa
}

\section{Effects of Non-Invasive Breathing Techniques on Improving Pulmonary Function in Cardiac Patients: An Integrative Review}

Danyele Holanda da Silva ${ }^{1 *}$, Tassiane Maria Alves Pereira², Janaína de Moraes Silva ${ }^{3}$

Como citar esse artigo. Silva, D. H; Pereira, T. M. A; Silva, J. M; Efeitos das Técnicas Respiratórias Não Invasivas na Melhora da Função Pulmonar de Cardiopatas: Uma Revisão Integrativa. Revista de Saúde 2021 Ago/Nov.; 12 (3): 26-32.

\begin{abstract}
Resumo
As doenças cardiovasculares (DCV) são a principal causa de morbimortalidade em países desenvolvidos e em desenvolvimento. De acordo com estimativas da Organização Mundial da Saúde, 17,9 milhões de pessoas morreram de DCV em 2016, representando 31\% de todas as mortes globais. Revisão integrativa realizada nas bases de dados PubMed, SciELO, Lilacs e PEDro, com base na questão norteadora da pesquisa, e cruzamento dos termos: exercícios respiratórios AND pré-operatório AND cirurgia cardíaca (todos os termos presentes em MEsh e DeCs) de maio a junho de 2020. Foram encontrados 104 artigos nas bases de dados selecionadas, 67 artigos foram excluídos, 37 foram selecionados para leitura completa, oito dos quais fizeram parte desta revisão. A presente revisão integrativa teve como objetivo analisar os efeitos das técnicas respiratórias na função pulmonar de pacientes submetidos à cirurgia cardíaca. Pacientes submetidos a procedimento cirúrgico cardíaco desenvolvem principalmente disfunção pulmonar pós-operatória com redução significativa dos volumes pulmonares, comprometimento da função respiratória, diminuição da complacência pulmonar e aumento do trabalho respiratório. Técnicas respiratórias como exercícios respiratórios e / ou treinamento muscular respiratório empregados em pacientes submetidos a procedimento cirúrgico cardíaco, melhoram a função pulmonar, conseqüentemente melhorando a força muscular respiratória como um todo, interferindo também na diminuição de complicações respiratórias pós-CRM nesta população.
\end{abstract}

Palavras-chave: Cirurgia cardíaca; Exercícios de respiração; Pré-operatório.

\section{Introdução}

As Doenças Cardiovasculares (DCV) constituem a principal causa de morbimortalidade nos países desenvolvidos e em desenvolvimento ${ }^{1}$. Segundo as estimativas da Organização Mundial da Saúde, 17,9 milhões de pessoas morreram de DCV em 2016, representando $31 \%$ de todas as mortes globais ${ }^{2}$. Elas podem estar relacionadas a fatores de risco como tabagismo, sedentarismo, obesidade, hipertensão, dieta inadequada, dislipidemia ${ }^{3}$, predisposição genética e fatores ambientais que podem ocorrer simultaneamente, caracterizando um maior risco comparado ao efeito isolado de cada fator ${ }^{4}$.

O tratamento das DCV, na maioria dos casos, está relacionado a realização de cirurgias, embora haja avanços nos procedimentos minimamente invasivos ampliando as possibilidades de tratamento, sendo as cirurgias de revascularização do miocárdio e valvares, os procedimentos cirúrgicos mais comuns. O implante de 
marcapasso cardíaco permanente (MP) tem aumentado nos últimos anos com a finalidade de tratar bradiarritmia, bloqueios atrioventriculares (BAV) (segundo grau ou total), doença do nó sinusal, fibrilação atrial ventricular de alta frequência e síndromes neuromediadas ${ }^{5,6}$.

As cirurgias cardíacas encontram-se entre os procedimentos cirúrgicos mais realizados no mundo, ocorrendo, em média, cerca de 2.000 nos Estados Unidos, 900 na Europa e, no Brasil, 350 a cada 1 milhão de habitantes por ano $^{7}$. Elas podem causar complicações que aumentam a morbimortalidade pós-operatória (PO), mesmo com o uso das melhores técnicas cirúrgicas e tratamento medicamentoso e fisioterapêutico, através do uso de anti-hipertensivos e hipolipemiantes e, programas de reabilitação cardíaca ${ }^{8,9,10}$. Dentre as principais, estão hipotensão, sangramento, hiperglicemia, insuficiência renal aguda, reoperação, tamponamento cardíaco, taquicardia ventricular, hipopotassemia, sepse, acidente vascular cerebral e parada cardiorrespiratória2, fraqueza muscular respiratória e periférica, diminuição da capacidade funcional, alterações pulmonares que incluem atelectasias, infecção respiratória e hipoxemia. Estas podem aumentar o tempo prolongado de internação hospitalar $^{11}$.

As técnicas respiratórias não invasivas são essenciais durante a internação hospitalar, tanto no pré quanto no pós-operatório visando prevenir os efeitos da imobilidade no leito, otimizar a independência funcional do paciente, reduzir complicações pulmonares mantendo as vias aéreas permeáveis, favorecendo uma ventilação adequada e o sucesso na extubação ${ }^{12,13}$.

Tendo em vista que as cirurgias cardíacas sejam um dos procedimentos mais realizados no mundo e os possíveis déficits pulmonaresquepodem estarassociados a essas cirurgias, acredita-se que as técnicas respiratórias não invasivas, tais como exercícios respiratórios e Treinamento Muscular Inspiratório (Threshold), exercícios respiratórios com associação de exercícios motores, possam melhorar a função pulmonar, e por sua vez, reduzir o índice de complicações pós-operatórias. Dessa forma, o objetivo do presente estudo foi analisar o efeito das técnicas respiratórias não invasivas na função pulmonar de pacientes submetidos a cirurgias cardíacas.

\section{Material e Métodos}

Este estudo trata-se de uma revisão integrativa conduzida de acordo com as diretrizes do Preferred Reporting Items for Systematic Reviews and MetaAnalyses (PRISMA) ${ }^{14}$, com utilização da estratégia PICO que, representa um acrônimo para População, Intervenção, Comparação e Desfecho $(\mathrm{P}=$ Pacientes elegíveis para cirurgias cardíacas; $\mathrm{I}=$ Técnicas respiratórias; $\mathrm{C}=$ não se aplica; $\mathrm{O}=$ Melhora da função pulmonar pós cirurgia cardíaca para formulação da pergunta norteadora da pesquisa e definição dos descritores ${ }^{15}$.

O levantamento dos artigos foi realizado nas bases PubMed, SciELO, Lilacs e PEDro, baseandose na pergunta norteadora da busca: "qual o efeito das técnicas respiratórias não-invasivas na função pulmonar de pacientes submetidos a cirurgias cardíacas?", e cruzamento dos termos: breathing exercises AND preoperative AND cardiac surgery (todos são termos presentes no MEsh e DeCs) no período de maio a junho de 2020. Foram incluídos artigos originais que utilizaram as técnicas respiratórias em pacientes elegíveis a cirurgia cardíaca, artigos nos idiomas inglês e português publicados nos últimos dez anos. Foram excluídos artigos com desenhos inapropriados, duplicados, que relacionaram a fisioterapia respiratória com outras patologias e que a realizassem somente no pós-operatório de cirurgias cardíacas.

\section{Resultados}

Foram encontrados 104 artigos nas bases de dados selecionadas, 67 artigos foram excluídos, selecionados 37, para a leitura na íntegra, sendo que oito compuseram esta revisão (Figura 1).

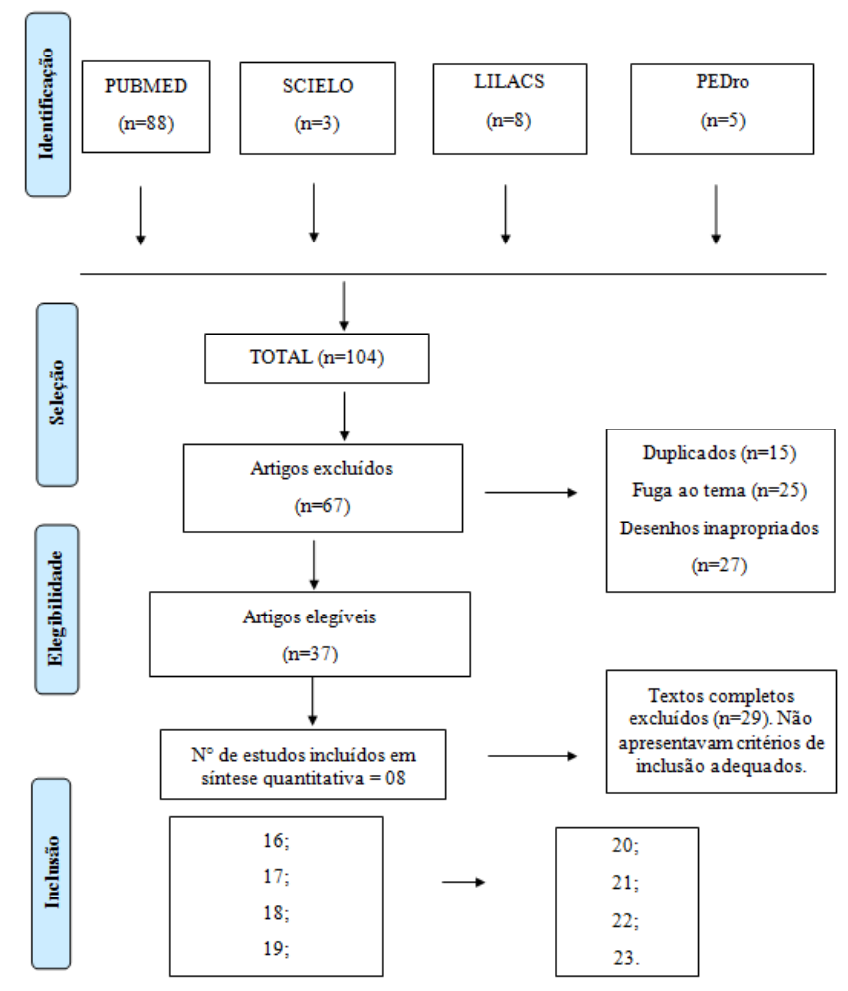

Figura 1. Fluxograma da estratégia de busca dos artigos.

Fonte: Autores, 2021 
Os artigos incluídos abordaram sobre a utilização de técnicas respiratórias em pacientes elegíveis a cirurgia cardíaca. Foram analisados 846 pacientes, com idade entre 18 anos e 80 anos, de ambos os sexos, sendo avaliado força muscular respiratória com utilização do manômetro ${ }^{17}$, método do pico do fluxo expiratório ${ }^{23}$, dispositivo eletrônico portátil de pressão bucal $^{16}$ e manuvacuometria ${ }^{22}$; capacidade funcional com Teste de Caminhada de seis minutos ${ }^{23,16}$; avaliação da função respiratória por meio da espirometria ${ }^{16,20,21,22}$, espirometria e gasometria ${ }^{18}$, método do pico do fluxo expiratório ${ }^{20}$; grau de dispneia (escala de borg) ${ }^{20}$; permanência hospitalar ${ }^{17,18,19,22}$; complicações pulmonares pós-operatórias ${ }^{19,22}$; análise da qualidade de vida $^{16,19}$; ansiedade e depressão ${ }^{16}$.

As intervenções utilizadas foram exercícios respiratórios e Treinamento Muscular Inspiratório

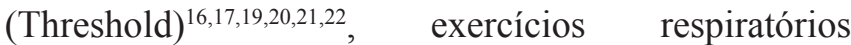
com associação de exercícios motores (exercícios respiratórios associado a exercícios de alongamento e relaxamento muscular de MMSS e MMII) $)^{16,17,18,23}$.

A pontuação adquirida entre os artigos selecionados nos resultados (quadro 1) através da escala PEDro, foram entre 5 e 9 . As produções incluídas foram apresentadas de acordo com a amostra apresentada abaixo (quadro 1).

Quadro 1. Caracterização do acervo de revisão.

\begin{tabular}{|c|c|c|c|c|c|}
\hline ESTUDO & AMOSTRA & INTERVENÇÃO & DESFECHO & RESULTADOS & $\begin{array}{c}\text { ESCALA } \\
\text { PEDRO }\end{array}$ \\
\hline 22 & $\begin{array}{l}197 \text { indiví- } \\
\text { duos com } \\
\text { idade } \geqslant \\
50 \text { anos pro- } \\
\text { gramados } \\
\text { para CRM. }\end{array}$ & $\begin{array}{l}\text { Grupo controle }(\mathrm{n}=99) \text { : Grupo } \\
\text { intervenção }(\mathrm{n}=98) \text {. Todos os } \\
\text { pacientes receberam os dois cui- } \\
\text { dados habituais (ou seja, educa- } \\
\text { ção, tosse e mobilização pre- } \\
\text { coce) e abdominal treinamento } \\
\text { respiratório antes da cirurgia até } \\
\text { a alta hospitalar. Ambos grupos } \\
\text { realizaram treinamento respira- } \\
\text { tório abdominal, duas vezes por } \\
\text { dia, com } 20 \text { minutos cada, nos } \\
\text { últimos cinco dias antes da data } \\
\text { real da cirurgia. O diafragma } \\
\text { músculos foram fortalecidos } \\
\text { através deste exercício. }\end{array}$ & $\begin{array}{l}\text { Incidência de Com- } \\
\text { plicações Pulmona- } \\
\text { res Pós-operatórias } \\
\text { (pneumopatia); As } \\
\text { variáveis de desfe- } \\
\text { cho secundárias fo- } \\
\text { ram força muscular } \\
\text { inspiratória, função } \\
\text { pulmonar e tempo de } \\
\text { internação. }\end{array}$ & $\begin{array}{l}\text { Em pacientes recebendo reabilitação } \\
\text { pré-operatória (grupo intervenção), a } \\
\text { incidência de complicações pulmona- } \\
\text { res pós-operatórias (pneumopatia) foi } \\
\text { reduzida em } 77 \% \text {, tendo aumento signi- } \\
\text { ficativo na força muscular inspiratória, } \\
\text { volume expiratório forçado no primeiro } \\
\text { segundo de expiração (VEF1) \% previs- } \\
\text { to, capacidade vital forçada (CVF) \% } \\
\text { prevista e ventilação voluntária máxima } \\
\text { (MVV) \% previsto. O tempo de interna- } \\
\text { ção foi de } 7,51 \text { dias para o mesmo e 9,38 } \\
\text { dias no grupo controle. }\end{array}$ & 9 \\
\hline 23 & $\begin{array}{l}59 \text { CRM, } \\
\text { idade mé- } \\
\text { dia: } 63,6 \pm \\
10,7 \text { anos, } \\
\text { a lo c a d o s } \\
\text { em: Grupo } \\
\text { A }(\mathrm{n}=19) ; \\
\text { Grupo B }(\mathrm{n} \\
=20) \text { e gru- } \\
\text { po C }(\mathrm{n}= \\
20) \text {. }\end{array}$ & $\begin{array}{l}\text { Grupo A (fisioterapia respirató- } \\
\text { ria e motora); grupo B (fisiotera- } \\
\text { pia respiratória e motora) e gru- } \\
\text { po C (grupo controle). Indicado } \\
\text { durante o período de educação } \\
\text { da fase pré-operatória, sendo re- } \\
\text { alizados diariamente para todos } \\
\text { os pacientes, geralmente por } 2 \\
\text { dias ou mais. Grupo C: auxilia- } \\
\text { dos apenas nas passagens pos- } \\
\text { turais e na caminhada assistida, } \\
\text { e suas condições clínicas foram } \\
\text { avaliadas diariamente. }\end{array}$ & $\begin{array}{l}\text { Capacidade respira- } \\
\text { tória, força muscular } \\
\text { e tolerância ao exer- } \\
\text { cício. }\end{array}$ & $\begin{array}{l}\text { Grupos A e B tiveram melhor capacidade } \\
\text { funcional (mais alta), menor tolerância } \\
\text { reduzida ao exercício e melhores valores } \\
\text { de PFE; houve uma baixa taxa de com- } \\
\text { plicações pulmonares pós-operatórias. } \\
\text { Diminuição da PaO } 2 \text { e SaO } 2 \text { observa- } \\
\text { dos após a cirurgia em comparação com } \\
\text { o período pré-operatório no grupo C. }\end{array}$ & 7 \\
\hline 20 & $\begin{array}{l}78 \text { pacien- } \\
\text { tes com e } \\
\text { sem DPOC } \\
\text { submetidos } \\
\text { à CRM, alo- } \\
\text { cados em: } \\
\text { grupo com } \\
\text { DPOC, n = } \\
40 ; \text { grupo } \\
\text { sem DPOC, } \\
\mathrm{n}=38 \text {. }\end{array}$ & $\begin{array}{l}\text { PRP: cessação do tabagismo, } \\
\text { exercícios respiratórios (3x/ } \\
\text { dia), exercícios para membros } \\
\text { superiores e inferiores ( } 30 \text { min/ } \\
\text { dia), espirometria de incentivo } \\
(3 \mathrm{x} / \text { dia }) \text {, pressão positiva inter- } \\
\text { mitente respiração (de } 10 \text { a } 15 \\
\text { min/dia) e fisioterapia respira- } \\
\text { tória (4x/dia). As intervenções } \\
\text { começaram } 1 \text { semana antes da } \\
\text { cirurgia e continuaram por } 2 \text { se- } \\
\text { manas no pós-operatório. }\end{array}$ & $\begin{array}{l}\text { Força muscular respi- } \\
\text { ratória e função pul- } \\
\text { monar. }\end{array}$ & $\begin{array}{l}\text { Aumento da CVF, VEF 1, PFE e FEF } \\
\text { no grupo com DPOC; Melhora da força } \\
\text { muscular respiratória no dia } 14 \text { pós-ope- } \\
\text { ratório em ambos os grupos, FR dimi- } \\
\text { nuiu nos dois grupos, sem diferença sig- } \\
\text { nificativa nas complicações pulmonares } \\
\text { (pneumonia, enfisema e atelectasia) e na } \\
\text { mortalidade entre eles. }\end{array}$ & 5 \\
\hline
\end{tabular}


Quadro 1 (cont.). Caracterização do acervo de revisão.

\begin{tabular}{|c|c|c|c|c|c|}
\hline ESTUDO & AMOSTRA & INTERVENÇÃO & DESFECHO & RESULTADOS & $\begin{array}{c}\text { ESCALA } \\
\text { PEDRO }\end{array}$ \\
\hline 21 & $\begin{array}{l}104 \text { CRM, } \\
\text { alocados em } \\
\text { dois grupos: } \\
52 \text { pacien- } \\
\text { tes interna- } \\
\text { dos (grupo } \\
\text { InPhysio) e } \\
52 \text { domici- } \\
\text { liares (grupo } \\
\text { home Phy- } \\
\text { sio). }\end{array}$ & $\begin{array}{l}\text { Grupo (InPhysio): cicloergôme- } \\
\text { tro (20/30 minutos); exercícios } \\
\text { coletivos de ginástica (20 min/ } \\
\text { dia); exercícios respiratórios } \\
\text { (10x/hora) por } 3 \text { semanas. Gru- } \\
\text { po (HomePhysio): exercícios } \\
\text { estacionários pré-agendados, } \\
\text { recomendações detalhadas de } \\
\text { reabilitação sobre a conduta fi- } \\
\text { sioterapêutica pós-operatória; } \\
\text { exercícios apropriados, sendo } \\
\text { cobertos por atendimento am- } \\
\text { bulatorial no Ambulatório de } \\
\text { Cirurgia Cardíaca. Realizaram } \\
\text { espirometria. }\end{array}$ & $\begin{array}{l}\text { O status da capacida- } \\
\text { de e função do siste- } \\
\text { ma respiratório }\end{array}$ & $\begin{array}{l}\text { Embora nenhum grupo tenha retornado } \\
\text { aos níveis pré-operatórios de CVF, a } \\
\text { vantagem da reabilitação hospitalar era } \\
\text { clara. As diferenças no VEF } 1.0 \text { final } \\
\text { não foram significativas entre os grupos, } \\
\text { mas grupo InPhysio haviam se recupera- } \\
\text { do para } 96 \% \text { da linha de base, enquanto } \\
\text { no grupo homePhysio eram de } 86 \% \text {. }\end{array}$ & 6 \\
\hline 19 & $\begin{array}{l}235 \text { pacien- } \\
\text { tes submeti- } \\
\text { dos a CRM. }\end{array}$ & $\begin{array}{l}\text { Grupo Intervenção (submetidos } \\
\text { a TMI; espirometria de incenti- } \\
\text { vo e educação sobre manobras } \\
\text { respiratórias profundas, tosse } \\
\text { e mobilização precoce no pré- } \\
\text { operatório) e grupo controle } \\
\text { (recebeu instruções sobre ma- } \\
\text { nobras de respiração profunda, } \\
\text { tosse e mobilização precoce no } \\
\text { dia anterior à cirurgia). Após a } \\
\text { cirurgia, os dois grupos rece- } \\
\text { beram incentivo espirometria e } \\
\text { fisioterapia respiratória, e todos } \\
\text { os pacientes seguiram o mesmo } \\
\text { esquema de mobilização. }\end{array}$ & $\begin{array}{l}\text { Desfecho primário: } \\
\text { complicações pulmo- } \\
\text { nares pós-operatórias } \\
\text { (pneumonia); Desfe- } \\
\text { cho secundário: Du- } \\
\text { ração do tempo de in- } \\
\text { ternação e qualidade } \\
\text { de vida. }\end{array}$ & $\begin{array}{l}\text { Complicações pulmonares pós-operató- } \\
\text { rias (pneumonia) e tempo de internação } \\
\text { diminuíram significativamente no grupo } \\
\text { de intervenção. Os cursos de tempo para } \\
\text { todos os pacientes mostraram melhorias } \\
\text { significativas na qualidade de vida após } \\
\text { cirurgia em comparação com a linha de } \\
\text { base. Não foram observadas diferenças } \\
\text { significativas na qualidade de vida ao } \\
\text { longo do tempo entre os dois grupos. }\end{array}$ & 8 \\
\hline 18 & $\begin{array}{l}60 \text { pacien- } \\
\text { tes CRM, } \\
39 \text { homens } \\
(65 \%) \text { e } 21 \\
\mathrm{~m} \text { u } 1 \text { h e r e s } \\
(35 \%) \text { com } \\
\text { idade média } \\
\text { de } 8,10 \pm \\
9,56, \text { divi- } \\
\text { didos em } 2 \\
\text { grupos: A e } \\
\text { B. }\end{array}$ & $\begin{array}{l}\text { Grupo A: fisioterapia pré-ope- } \\
\text { ratória com foco no fortaleci- } \\
\text { mento dos músculos inspirató- } \\
\text { rios e cavidade torácica sendo } \\
\text { realizada com base nas rotinas } \\
\text { da enfermaria durante } 15 \text { dias, } \\
25 \text { minutos cada sessão. Grupo } \\
\text { B: fisioterapia pós-operatória: } \\
\text { Técnicas para limpar os pul- } \\
\text { mões, incluindo mobilização, } \\
\text { técnicas manuais, uso do ciclo } \\
\text { ativo de técnicas respiratórias } \\
\text { e uso de SI; movimentos ativos } \\
\text { auxiliares das extremidades; } \\
\text { movimento ativo das extremi- } \\
\text { dades; Exercícios respiratórios } \\
\text { e expansão dos lobos dos pul- } \\
\text { mões. }\end{array}$ & $\begin{array}{l}\text { Tempo de permanên- } \\
\text { cia na UTI, o tempo } \\
\text { de uso da VM, função } \\
\text { respiratória. }\end{array}$ & $\begin{array}{l}\text { A diferença nas médias de CVF, PFE e } \\
\text { PCO } 2 \text { foi maior no grupo A (interven- } \\
\text { ção); diminuição no tempo de VM em } \\
\text { uso e internado em UTI. }\end{array}$ & 7 \\
\hline 17 & $\begin{array}{l}70 \text { pacien- } \\
\text { tes CRM, } \\
\text { de ambos os } \\
\text { sexos, com } \\
\text { idades entre } \\
40 \text { e } 75 \text { anos }\end{array}$ & $\begin{array}{l}\text { Grupo I (GI) -35 pacientes, sub- } \\
\text { metidos a intervenção terapêu- } \\
\text { tica, uma vez ao dia, no período } \\
\text { que antecedeu a cirurgia (exer- } \\
\text { cícios respiratórios com asso- } \\
\text { ciação do TMI e Grupo II (GII) } \\
\text { - } 35 \text { pacientes que não foram } \\
\text { submetidos protocolo de fisiote- } \\
\text { rapia no período pré-operatório, } \\
\text { recebendo apenas orientações } \\
\text { da rotina de enfermaria. }\end{array}$ & $\begin{array}{l}\text { Tempo da permanên- } \\
\text { cia hospitalar, altera- } \\
\text { ções nos volumes pul- } \\
\text { monares (capacidade } \\
\text { respiratória) e força } \\
\text { muscular respiratória }\end{array}$ & $\begin{array}{l}\text { Pressão inspiratória máxima no terceiro } \\
\text { e quinto dia pós-operatório dia e diferen- } \\
\text { ça significativa entre grupos, sendo me- } \\
\text { lhor para o grupo de intervenção. Pres- } \\
\text { são expiratória muscular foi significativa } \\
\text { no quinto dia de pós-operatório no grupo } \\
\text { intervenção comparado aos controles. A } \\
\text { diferença de tempo de permanência de } \\
\text { hospitalização no pós-operatório foi } \\
\text { encontrada entre os grupos com menor } \\
\text { internação no grupo que recebeu terapia } \\
\text { pré-operatória. }\end{array}$ & 7 \\
\hline
\end{tabular}


Quadro 1 (cont.). Caracterização do acervo de revisão.

\begin{tabular}{|c|c|c|c|c|c|}
\hline ESTUDO & AMOSTRA & INTERVENÇÃO & DESFECHO & RESULTADOS & $\begin{array}{l}\text { ESCALA } \\
\text { PEDRO }\end{array}$ \\
\hline 16 & $\begin{array}{l}43 \text { pacientes } \\
\text { submetidos } \\
\text { à CRM, di- } \\
\text { vididos em } \\
\text { grupo de } \\
\text { intervenção } \\
(\mathrm{n}=22) \text { e do } \\
\text { grupo con- } \\
\text { trole }(\mathrm{n}=21) .\end{array}$ & $\begin{array}{l}\text { Os indivíduos de ambos os gru- } \\
\text { pos receberam os mesmos cui- } \\
\text { dados uma vez por dia. Grupo } \\
\text { intervenção: mobilização com } \\
\text { exercícios ativos dos membros } \\
\text { superiores e inferiores, tórax, } \\
\text { exercícios respiratórios e técni- } \\
\text { cas de tosse; e TMI, cinco dias } \\
\text { no pré-operatório, cinco dias no } \\
\text { pós-operatório período. Gru- } \\
\text { po controle: mobilização com } \\
\text { exercícios ativos dos membros } \\
\text { superiores e inferiores, tórax, } \\
\text { exercícios respiratórios e técni- } \\
\text { cas de tosse, quinto dia de pós- } \\
\text { operatório. }\end{array}$ & $\begin{array}{l}\text { Função e força mus- } \\
\text { cular respiratória; ca- } \\
\text { pacidade funcional; } \\
\text { qualidade de vida; A } \\
\text { ansiedade e a depres- } \\
\text { são. }\end{array}$ & $\begin{array}{l}\text { A força muscular inspiratória média au- } \\
\text { mentou, a melhora na qualidade de vida } \\
\text { para a dimensão do sono foi maior no } \\
\text { grupo intervenção, como percorreram } \\
\text { uma distância maior durante o TC6 do } \\
\text { que os cuidados usuais. Os escores de } \\
\text { ansiedade foram significativamente e } \\
\text { tempo de permanência na UTI foram } \\
\text { menores no grupo de intervenção do que } \\
\text { no grupo de atendimento habitual. }\end{array}$ & 8 \\
\hline
\end{tabular}

Legenda. CRM (Cirurgia de Revascularização Miocárdica); UTI (Unidade de Terapia Intensiva); VM (ventilação Mecânica); CVF (Capacidade Vital Forçada); PFE (Pico de Fluxo Expiratório); PCO 2 (pressão arterial parcial de dióxido de carbono); GRC (Grupo de Reabilitação Cardíaca); TMI (treinamento Muscular Inspiratório); QV (Qualidade de vida); GC (Grupo Controle); GT (Grupo Treinamento); DTC6 (Distância do Teste de Caminhada de 6 minutos); VO 2 pico (volume de oxigênio); GI (Grupo Intervenção); CPP (Complicações Pulmonares Pós-operatórias); TC6 (teste de Caminhada de "minutos); PRP (Programa de Reabilitação Pulmonar); VEF (Volume Expiratório Final); VEF 1 (Volume Expiratório Final no primeiro minuto); FEF (Fluxo Expiratório Forçado); DPOC (Doença Pulmonar Obstrutiva Crônica); FR (Frequência Respiratória); GAE (Grupo de exercícios aeróbicos); PImáx (Pressão Inspiratória máxima); PaO 2 (Pressão Parcial de oxigênio); $\mathrm{SaO} 2$ (Saturação de Oxigênio).

Fonte: Autores, 2021.

\section{Discussão}

A presente revisão integrativa teve como objetivo analisar os efeitos das técnicas respiratórias não invasivas na função pulmonar de pacientes submetidos a cirurgias cardíacas.

Pacientes submetidos a algum procedimento cirúrgico cardíaco, desenvolvem principalmente disfunção pulmonar pós-operatória com redução significativa dos volumes pulmonares, comprometimento da função respiratória, diminuição da complacência pulmonar e aumento do trabalho respiratório. A redução dos volumes e capacidades pulmonares contribui para alterações nas trocas gasosas, resultando em hipoxemia ${ }^{17,20}$, podem apresentar também comprometimento funcional pós-operatório, comprometendo significativamente sua capacidade de recuperação e nível de autonomia e geralmente levando à redução da tolerância ao exercício relacionado à esternotomia, à circulação extracorpórea e redução da atividade física, logo programas de treinamento baseados em fisioterapia respiratória e musculoesquelética tornam-se assim a pedra angular para uma melhor recuperação pós-operatória ${ }^{23}$.

Os estudos utilizaram técnicas respiratórias não invasivas como o Treinamento Muscular Respiratório $(\mathrm{TMI})^{22,20,21,19,17,16}$ e exercícios respiratórios com associação de exercícios motores (associação de exercícios de alongamento e relaxamento muscular de MMSS e MMII) $)^{23,17,16}$, evidenciando melhoras na força muscular respiratória, na função pulmonar e reduções de complicações pulmonares pós-operatórias.

O TMI trata-se de um treinamento inspiratório baseado em dispositivos que permitem pressões específicas e consistentes para melhorar a força da musculatura respiratória, independente do fluxo que o paciente realiza durante a respiração ${ }^{24}$, ou seja, a melhora da força muscular respiratória, se dá pelos princípios de treinamento, sobrecarga, na qual o músculo deve ser solicitado em níveis superiores ao habitual, para que as células musculares aumentem de tamanho ou capacidade funcional e especificidade, onde o treinamento deve ser direcionado especificamente às propriedades musculares, que no qual, melhora a vasodilatação sistêmica e a perfusão dos músculos periféricos ${ }^{20,25,26,27}$.

De fato, é possível que qualquer estímulo ao treinamento, independentemente da especificidade dos músculos respiratórios, possa melhorar a força muscular respiratória e diminuir sintomas relacionados, como dispneia e intolerância ao exercício, tendo assim, relação com uma boa recuperação destes pacientes ${ }^{28}$.

As técnicas de fisioterapia da cavidade torácica são utilizadas com o objetivo de aumentar os volumes respiratórios, melhorar a oxigenação e diminuir as complicações respiratórias após a CRM, e o exercício físico é um componente primordial num programa de 
reabilitação cardíaca. A associação do treinamento físico aeróbio mostra uma melhora cardiovascular, muscular esquelética, resistência, função pulmonar, perfil inflamatório, melhora na qualidade de vida, além de melhorar resultados significativos como mortalidade ${ }^{25}$. Grande parte do sucesso desses programas se deve à terapia baseada neste, que proporciona ao paciente maior capacidade de recuperação, permitindo o retorno às suas atividades habituais levando estes a participar de suas atividades da vida diária de forma mais independente possível $^{2,16,18,20,21}$.

As limitações presentes neste estudo estão relacionadas com o número reduzido de pesquisas científicas sobre intervenções da fisioterapia respiratória especificamente no período pré-operatório de pacientes em procedimentos cirúrgicos cardíacos, logo nota-se a importância e necessidade de mais estudos voltados ao período pré-intervenção cirúrgica cardíaca, para que se possa elucidar evidências positivas sobre a utilização de técnicas respiratórias pré-cirúrgicas que contribuam principalmente para a redução de possíveis complicações após a cirurgia.

\section{Considerações Finais}

Diante dos achados científicos discutidos na presente revisão integrativa, conclui-se que as técnicas respiratórias não invasivas tais como exercícios respiratórios e/ou Treinamento Muscular Respiratório empregadas em pacientes submetidos a algum procedimento cirúrgico cardíaco, melhoram a função pulmonar, consequentemente melhora a força muscular respiratória como um todo, interferindo também em diminuição de complicações respiratórias pós-CRM nesta população.

\section{Referências}

1. Rodrigues HF, Furuya RK, Dantas RAS, Dessotte CAM. Anxiety and depression in cardiac surgery: sex and age range differences. Esc Anna Nery - Rev Enferm. 2016;20(3):1-7.

2. Oliveira R, Lopes P, Soares C, Nogueira C, Braga DV, Gomes JR. Complicações do pós-operatório imediato de cirurgia cardíaca eletiva: estudo transversal à luz de Roy. 2019;23-32.

3. Garcia GD, Pompeo DA, Eid LP, Cesarino CB, Pinto MH, Gonçalves LWP. Relationship between anxiety, depressive symptoms and compulsive overeating disorder in patients with cardiovascular diseases. Rev Lat Am Enfermagem. 2018;26:1-9.

4. Teston EF, Cecilio HPM, Santos AL, De Arruda GO, Radovanovic CAT, Marcon SS. Fatores associados às doenças cardiovasculares em adultos. Med. 2016;49(2):95-102.

5. Dessotte CAM, Furuya RK, Rodrigues HF, Rossi LA, Dantas RAS. Relation between stressors and hemodynamic instability in the postoperative period after cardiac surgery. Texto e Context Enferm. 2018;27(3):1-9.

6. Pesente TS, Freitas TLL de, Korb JP, Ferreira JN, Dias AS, Sachetti A. Pacemaker implications in the functional capacity and quality of life of cardiopathic patients. Fisioter em Mov. 2019;32:1-9.

7. Braz N de J, Evangelista S de S, Evangelista S de S, Garbaccio JL, Oliveira AC de. Retrospective or clue-based surveillance? What is the impact on the notification of surgical site infections in cardiac surgeries? Texto e Context Enferm. 2018;27(4):1-6.

8. Rodrigues HF, Furuya RK, Dantas RAS, Rodrigues AJ, Dessotte CAM. Association of preoperative anxiety and depression symptoms with postoperative complications of cardiac surgeries. Rev Lat Am Enfermagem. 2018;26:1-10

9. Luymes CH, Poortvliet RKE, van Geloven N, de Waal MWM, Drewes YM, Blom JW, et al. Deprescribing preventive cardiovascular medication in patients with predicted low cardiovascular disease risk in general practice the ECSTATIC study: A cluster randomised non-inferiority trial. BMC Med. 2018;16(1):1-14. https://doi.org/10.1186/s12916-017-0988-0.

10. Lemos M, Torres S, Jaramillo I, Gómez PE, Barbosa A. Percepciones de la enfermedad y hábitos de vida saludable en personas con enfermedads crónicas. Psicogente. 2019;22(42):1-20.

11. Cordeiro ALL, Borges DL, Peruna MP, Guimarães AR, Cacau L de A. Correlação entre a Duração da Internação Hospitalar e a Velocidade da Marcha em Pacientes Submetidos à Cirurgia Cardíaca. Int J Cardiovasc Sci. 2017;30:123-127

12. Gomes AO, Ramos WR, Dalfior C dos A, Cavalcante MG, De Abreu I, Abrão FC. Protocolo fisioterapêutico aplicado no pós-operatório imediato para recuperação acelerada de pacientes submetidos à procedimentos cirúrgicos torácicos no Hospital Santa Marcelina - Itaquera (PROSM): estudo clínico randomizado. Rev Pesq em Fisioter. 2018;8(2):279-86.

13. Strolischein CAH, Silva DR da, Costa EL, Sancoré1 FD, Fontoura2 TGKAFC. Prevalência das principais complicações pós-operatórias em cirurgias cardíacas de revascularização do miocárdio em hospital filantrópico de Cuiabá-MT. J Chem Inf Model. 2019;53(9):1689-99.

14. Roncada C, Medeiros TM, Strassburger MJ, Strassburger SZ. Comparação da qualidade de vida relacionada à saúde de crianças / adolescentes com asma e de seus cuidadores: uma revisão sistemática e meta-análise. J Bras Pneumol. 2020;46(3):1-10.

15. Mairot LT da S, Costa BBG da, Heringer TPM, Borges RC, Moura EP. As Artes na Educação Médica: Revisão Sistemática da Literatura Arts in Medical Education: a Systematic Literature Review. Rev Bras Educ Med. 2019;43(4):54-64. http://dx.doi.org/10.1590/1981$52712015 \mathrm{v} 43 \mathrm{n} 4 \mathrm{RB} 20180146$.

16. Savci S, Degirmenci B, Saglam M, Arikan H, Inal-Ince D, Turan $\mathrm{HN}$, et al. Short-term effects of inspiratory muscle training in coronary artery bypass graft surgery: A randomized controlled trial. Scand Cardiovasc J. 2011;45(5):286-93.

17. Sobrinho MT, Guirado GN, Silva MA de M. Fisioterapia préoperatória restabelece parâmetros ventilatórios e reduz tempo de internação após revascularização do miocárdio. Brazilian J Cardiovasc Surg. 2014;29(2):221-8.

18. Shakuri SK, Salekzamani Y, Taghizadieh A, Sabbagh-Jadid H, Soleymani J, Sahebi L, et al. Effect of Respiratory Rehabilitation before Open Cardiac Surgery on Respiratory Function: A Randomized Clinical Trial. J Cardiovasc Thorac Res. 2015;7(1):13-7.

19. Valkenet K, Trappenburg JCA, Hulzebos EH, van Meeteren NLU, Backx FJG. Effects of a pre-operative home-based inspiratory muscle training programme on perceived health-related quality of life in patients undergoing coronary artery bypass graft surgery. Physiother (United Kingdom) [Internet]. 2017;103(3):276-82

20. Chen JO, Liu JF, Liu YQ, Chen YM, Tu ML, Yu HR, et al. Effectiveness of a perioperative pulmonary rehabilitation program following coronary artery bypass graft surgery in patients with and without COPD. Int J COPD. 2018;13:1591-7.

21. Szylińska A, Listewnik M, Rotter I, Rył A, Kotfis K, Mokrzycki K, et al. The efficacy of inpatient vs. Home-based physiotherapy following coronary artery bypass grafting. Int J Environ Res Public Health. 2018;15(11):1-11.

22. Chen X, Hou L, Zhang Y, Liu X, Shao B, Yuan B, et al. The effects of five days of intensive preoperative inspiratory muscle training on postoperative complications and outcome in patients having cardiac surgery: 
a randomized controlled trial. Clin Rehabil. 2019;33(5):913-22.

23. Nardi P, Pellegrino A, Pisano C, Vacirca SR, Anselmi D, Saulle $\mathrm{S}$, et al. The effect of preoperative respiratory physiotherapy and motor exercise in patients undergoing elective cardiac surgery: Short-term results. Kardiochirurgia i Torakochirurgia Pol. 2019;16(2):81-7.

24. Moreira AM, Galvão MLC, de Araújo HAG, Silva AT, Dos Reis LM, Silva AM, et al. Treino muscular respiratório e terapia vibratória em pacientes com doença de Parkinson. Rev Neurociencias. 2015;23(4):479-85.

25. Miozzo AP, Stein C, Marcolino MZ, Sisto IR, Hauck M, Coronel CC, et al. Effects of high-intensity inspiratory muscle training associated with aerobic exercise in patients undergoing CABG: Randomized clinical trial. Brazilian J Cardiovasc Surg. 2018;33(4):376-83.

26. Cordeiro ALL, de Melo TA, Neves D, Luna J, Esquivel MS, Guimarães $\mathrm{ARF}$, et al. Inspiratory muscle training and functional capacity in patients undergoing cardiac surgery. Brazilian J Cardiovasc Surg. 2016;31(2):140-4.

27. Hermes BM, Cardoso DM, Gomes TJN, dos Santos TD, Vicente MS, Pereira SN, et al. Treinamento muscular inspiratório de curto prazo potencializa os benefícios do treinamento aeróbico e resistido em pacientes após CRM na fase II de programa de reabilitação cardíaca. Brazilian J Cardiovasc Surg. 2015;30(4):474-81.

28. Mediano MFF, Mendes F de SNS, Pinto VLM, Da Silva GMS, Da Silva PS, Carneiro FM, et al. Cardiac rehabilitation program in patients with chagas heart failure: A single-arm pilot study. Rev Soc Bras Med Trop. 2016;49(3):319-28 